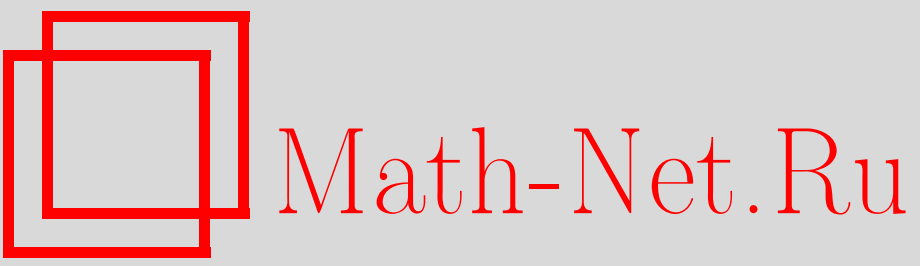

П. А. Крутицкий, В. В. Колыбасова, О смешанной задаче для уравнения Гельмгольца в плоской области с разрезами, УМH, 2005, том 60, выпуск 5, 167-168

DOI: https://doi.org/10.4213/rm1647

Использование Общероссийского математического портала Math-Net.Ru подразумевает, что вы прочитали и согласны с пользовательским соглашением

http://www.mathnet.ru/rus/agreement

Параметры загрузки:

IP: 54.92 .164 .108

26 апреля 2023 г., 15:09:20

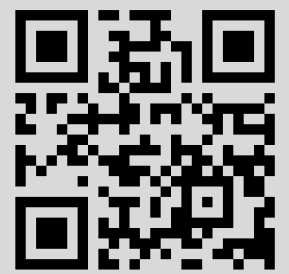




\title{
О СМЕШАННОЙ ЗАДАЧЕ ДЛЯ УРАВНЕНИЯ ГЕЛЬМГОЛЬЦА В ПЛОСКОЙ ОБЛАСТИ С РАЗРЕЗАМИ
}

\author{
П. А. Крутицкий, В.В.КолыБАСОвА
}

На плоскости $x \in \mathbb{R}^{2}$ рассмотрим многосвязную область, ограниченную простыми разомкнутыми кривыми $\Gamma_{1}^{1}, \ldots, \Gamma_{N_{1}}^{1} \in C^{2, \lambda}$ и простыми замкнутыми кривыми $\Gamma_{1}^{2}, \ldots, \Gamma_{N_{2}}^{2} \in C^{2, \lambda}, \lambda \in$ $(0,1]$, так, что кривые не имеют общих точек, в частности, концов. Будем рассматривать как случай внешней области, так и случай внутренней области, когда кривая $\Gamma_{1}^{2}$ охватывает все остальные. Положим $\Gamma^{1}=\bigcup_{n=1}^{N_{1}} \Gamma_{n}^{1}, \Gamma^{2}=\bigcup_{n=1}^{N_{2}} \Gamma_{n}^{2}, \Gamma=\Gamma^{1} \cup \Gamma^{2}$. Связную область, ограниченную $\Gamma^{2}$ и содержащую $\Gamma^{1}$, будем называть $\mathscr{D}$, так что $\partial \mathscr{D}=\Gamma^{2}, \Gamma^{1} \subset \mathscr{D}$. Предположим, что каждая кривая $\Gamma_{n}^{j}$ параметризована длиной дуги $s: \Gamma_{n}^{j}=\left\{x: x=x(s)=\left(x_{1}(s), x_{2}(s)\right), s \in\left[a_{n}^{j}, b_{n}^{j}\right]\right\}$, $n=1, \ldots, N_{j}, j=1,2$, так, что $a_{1}^{1}<b_{1}^{1}<\cdots<a_{N_{1}}^{1}<b_{N_{1}}^{1}<a_{1}^{2}<b_{1}^{2}<\cdots<a_{N_{2}}^{2}<b_{N_{2}}^{2}$ и область $\mathscr{D}$ остается справа при возрастании параметра $s$ на $\Gamma_{n}^{2}$. Совокупности отрезков оси $O s$ $\bigcup_{n=1}^{N_{1}}\left[a_{n}^{1}, b_{n}^{1}\right], \bigcup_{n=1}^{N_{2}}\left[a_{n}^{2}, b_{n}^{2}\right]$ и $\bigcup_{j=1}^{2} \bigcup_{n=1}^{N_{j}}\left[a_{n}^{j}, b_{n}^{j}\right]$ далее также будем обозначать $\Gamma^{1}, \Gamma^{2}$ и $\Gamma$ соответственно. Положим $C^{j, r}\left(\Gamma_{n}^{2}\right)=\left\{\mathscr{F}(s): \mathscr{F}(s) \in C^{j, r}\left[a_{n}^{2}, b_{n}^{2}\right], \mathscr{F}^{(m)}\left(a_{n}^{2}\right)=\mathscr{F}^{(m)}\left(b_{n}^{2}\right)\right.$, $m=0, j\}, j=0,1, r \in[0,1]$, и $C^{j, r}\left(\Gamma^{2}\right)=\bigcap_{n=1}^{N_{2}} C^{j, r}\left(\Gamma_{n}^{2}\right)$. Пусть $\mathbf{n}_{x}=\left(x_{2}^{\prime}(s),-x_{1}^{\prime}(s)\right)-$ вектор нормали к $\Gamma$ в точке $x(s)$. Будем рассматривать $\Gamma^{1}$ как совокупность разрезов. Будем говорить, что функция $u(x)$ принадлежит классу гладкости $\mathbf{K}$, если:

(а) $u \in C^{0}\left(\overline{\mathscr{D} \backslash \Gamma^{1}}\right) \cap C^{2}\left(\mathscr{D} \backslash \Gamma^{1}\right)$ и $u(x)$ непрерывна на концах разрезов $\Gamma^{1}$;

(б) $\nabla u \in C^{0}\left(\overline{\mathscr{D} \backslash \Gamma^{1}} \backslash X\right)$, где $X$-множество точек, состоящее из концов $\Gamma^{1}: X=\bigcup_{n=1}^{N_{1}}\left(x\left(a_{n}^{1}\right)\right.$ $\left.\cup x\left(b_{n}^{1}\right)\right)$;

(в) в окрестности любой точки $x(d) \in X$ для некоторых констант $\mathscr{C}>0, \varepsilon>-1$ выполняется неравенство $|\nabla u| \leqslant \mathscr{C}|x-x(d)|^{\varepsilon}$, где $x \rightarrow x(d)$ и $d=a_{n}^{1}$ или $d=b_{n}^{1}, n=1, \ldots, N_{1}$.

В определении класса $\mathbf{K} u(x)$ и $\nabla u(x)$ непрерьвно продолжимы на разрезы $\Gamma^{1} \backslash X$ слева и справа, но могут иметь скачок при переходе через $\Gamma^{1} \backslash X$.

ЗАДАчА $\mathbf{U}$. Найти функцию $u(x)$ из класса $\mathbf{K}$, которая удовлетворяет уравнению Гельмгольца $u_{x_{1} x_{1}}(x)+u_{x_{2} x_{2}}(x)+k^{2} u(x)=0, x \in \mathscr{D} \backslash \Gamma^{1}, k=$ const, $\operatorname{Im} k>0$, и граничным условиям: $\left.\left(\partial u(x) / \partial \mathbf{n}_{x}\right)\right|_{x(s) \in \Gamma^{1}}=f_{1}(s) \in C^{0, \lambda}\left(\Gamma^{1}\right),\left.u(x)\right|_{x(s) \in \Gamma^{2}}=f_{2}(s) \in C^{1, \lambda}\left(\Gamma^{2}\right), \lambda \in(0,1]$. Если $\mathscr{D}$-внешняя область, добавим условия на бесконечности: $u=o\left(|x|^{-1 / 2}\right),|\nabla u(x)|=o\left(|x|^{-1 / 2}\right)$, $|x|=\sqrt{x_{1}^{2}+x_{2}^{2}} \rightarrow \infty$.

Теорема 1. Если $\Gamma \in C^{2, \lambda}, \lambda \in(0,1]$, то задача $\mathbf{U}$ имеет не более одного решения. Будем искать решение задачи $\mathbf{U}$ в виде

$$
u[\mu](x)=\frac{i}{4} \int_{\Gamma^{1}} \mu(\sigma) V(x, \sigma) d \sigma+\frac{i}{4} \int_{\Gamma^{2}} \mu(\sigma) \frac{\partial \mathscr{H}_{0}^{(1)}(k|x-y(\sigma)|)}{\partial \mathbf{n}_{y}} d \sigma,
$$

где $V(x, \sigma)=\int_{a_{n}^{1}}^{\sigma} \frac{\partial \mathscr{H}_{0}^{(1)}(k|x-y(\xi)|)}{\partial \mathbf{n}_{y}} d \xi, \sigma \in\left[a_{n}^{1}, b_{n}^{1}\right], n=1, \ldots, N_{1}, H_{0}^{(1)}(z)-$ функция Ханкеля первого рода нулевого порядка. Будем искать $\mu(s)$ в пространстве $C_{q}^{\omega}\left(\Gamma^{1}\right) \cap C^{1, \lambda / 4}\left(\Gamma^{2}\right)$, $\omega \in(0,1], q \in[0,1)$, с нормой $\|\cdot\|_{C_{q}^{\omega}\left(\Gamma^{1}\right)}+\|\cdot\|_{C^{1, \lambda / 4}\left(\Gamma^{2}\right)}$. Будем говорить, что $\mu(s) \in$ $C_{q}^{\omega}\left(\Gamma^{1}\right)$, если $\mu_{1}(s) \in C^{0, \omega}\left(\Gamma^{1}\right)$, где $\mu_{1}(s)=\mu(s) \prod_{n=1}^{N_{1}}\left|s-a_{n}^{1}\right|^{q}\left|s-b_{n}^{1}\right|^{q}$, и $\|\mu(\cdot)\|_{C_{q}^{\omega}\left(\Gamma^{1}\right)}=$ $\left\|\mu_{1}(\cdot)\right\|_{C^{0, \omega}\left(\Gamma^{1}\right)}$. Кроме того, функция $\mu(s)$ должна удовлетворять условиям

$$
\int_{a_{n}^{1}}^{b_{n}^{1}} \mu(\sigma) d \sigma=0, \quad n=1, \ldots, N_{1}
$$

Работа выполнена при поддержке РФФИ (грант № 05-01-00050). 
Можно проверить, что если $\mu(s)$ принадлежит пространству $C_{q}^{\omega}\left(\Gamma^{1}\right) \cap C^{1, \lambda / 4}\left(\Gamma^{2}\right), \omega \in(0,1]$, $q \in[0,1)$, и удовлетворяет условиям (2), то функция (1) удовлетворяет всем условиям задачи $\mathbf{U}$, кроме граничных условий. Чтобы удовлетворить граничным условиям, подставим в них функцию (1) и получим интегральные уравнения для $\mu(s)$ на $\Gamma^{1}$ и $\Gamma^{2}$ :

$$
\begin{gathered}
\frac{1}{\pi} \int_{\Gamma^{1}} \frac{\mu(\sigma) d \sigma}{\sigma-s}+\int_{\Gamma} \mu(\sigma) Y_{1}(s, \sigma) d \sigma=-2 f_{1}(s), \quad s \in \Gamma^{1}, \\
\mu(s)+\int_{\Gamma} \mu(\sigma) Y_{2}(s, \sigma) d \sigma=2 f_{2}(s), \quad s \in \Gamma^{2},
\end{gathered}
$$

где

$$
\begin{aligned}
Y_{1}(s, \sigma)= & \left\{(1-\delta(\sigma))\left[\frac{1}{\pi}\left(\frac{\sin \varphi_{0}(x(s), y(\sigma))}{|x(s)-y(\sigma)|}-\frac{1}{\sigma-s}\right)-\frac{i}{2} \frac{\partial}{\partial \mathbf{n}_{x}} V_{0}(x(s), \sigma)\right]\right. \\
& \left.-\frac{i}{2} \delta(\sigma) \frac{\partial}{\partial \mathbf{n}_{x}} \frac{\partial}{\partial \mathbf{n}_{y}} \mathscr{H}_{0}^{(1)}(k|x(s)-y(\sigma)|)\right\} \in C^{0, p_{0}}\left(\Gamma^{1} \times \Gamma\right),
\end{aligned}
$$

$p_{0}=\lambda$, если $0<\lambda<1$, и $p_{0}=1-\varepsilon_{0}$ для любого $\varepsilon_{0} \in(0,1)$, если $\lambda=1 ; \delta(s)=0$, если $s \in \Gamma^{1}$, и $\delta(s)=1$, если $s \in \Gamma^{2}$;

$$
\begin{gathered}
V_{0}(x, \sigma)=\int_{a_{n}^{1}}^{\sigma} \frac{\partial h(k|x-y(\xi)|)}{\partial \mathbf{n}_{y}} d \xi, \quad \sigma \in\left[a_{n}^{1}, b_{n}^{1}\right], n=1,2, \ldots, N_{1}, h(z)=\mathscr{H}_{0}^{(1)}(z)-\frac{2 i}{\pi} \ln \frac{z}{k} ; \\
Y_{2}(s, \sigma)=\left\{\frac{i}{2}(1-\delta(\sigma)) V(x(s), \sigma)+\frac{i}{2} \delta(\sigma) \frac{\partial}{\partial \mathbf{n}_{y}} \mathscr{H}_{0}^{(1)}(k|x(s)-y(\sigma)|)\right\} \in C^{0}\left(\Gamma^{2} \times \Gamma\right) .
\end{gathered}
$$

Через $\varphi_{0}(x, y)$ обозначен угол между вектором $\overrightarrow{x y}$ и направлением нормали $\mathbf{n}_{x}$. Угол $\varphi_{0}(x, y)$ считается положительным, если он отложен от $\mathbf{n}_{x}$ против часовой стрелки, и отрицательньп в противном случае. Кроме того, $\varphi_{0}(x, y)$ непрерьвен при $x, y \in \Gamma$, если $x \neq y$.

Учитьвая гладкость кривых $\Gamma^{2}$ и функции $f_{2}(s)$, можно показать, что всякое решение уравнения (3b) в пространстве $C_{q}^{\omega}\left(\Gamma^{1}\right) \cap C^{0}\left(\Gamma^{2}\right), \omega \in(0,1], q \in[0,1)$, автоматически принадлежит $C_{q}^{\omega}\left(\Gamma^{1}\right) \cap C^{1, \lambda / 4}\left(\Gamma^{2}\right)$. Поэтому ниже будем искать решение системы $(2),(3)$ в $C_{q}^{\omega}\left(\Gamma^{1}\right) \cap C^{0}\left(\Gamma^{2}\right)$. $\mathrm{C}$ помощью теоремы 1 можно доказать, что однородная система $(2),(3)$ имеет только тривиальное решение в пространстве $C_{q}^{\omega}\left(\Gamma^{1}\right) \cap C^{0}\left(\Gamma^{2}\right), \omega \in(0,1], q \in[0,1)$. Используя этот факт и следствие 1 в [1], можно доказать следующую лемму.

Лемма. Если $\Gamma \in C^{2, \lambda}, f_{1}(s) \in C^{0, \lambda}\left(\Gamma^{1}\right), f_{2}(s) \in C^{1, \lambda}\left(\Gamma^{2}\right), \lambda \in(0,1]$, mo cucmeма уравнений (2), (3) имеет решение $\mu(s) \in C_{1 / 2}^{p}\left(\Gamma^{1}\right) \cap C^{0}\left(\Gamma^{2}\right), p=\min \{1 / 2, \lambda\}$. Это решение системь (2), (3) единственно в пространстве $C_{1 / 2}^{p}\left(\Gamma^{1}\right) \cap C^{0}\left(\Gamma^{2}\right)$.

Согласно сказанному выше, решение системы (2), (3), гарантированное леммой, автоматически принадлежит $C_{1 / 2}^{p}\left(\Gamma^{1}\right) \cap C^{1, \lambda / 4}\left(\Gamma^{2}\right)$. Из приведенных рассуждений вытекает

Tеорема 2. Если $\Gamma \in C^{2, \lambda}, f_{1}(s) \in C^{0, \lambda}\left(\Gamma^{1}\right), f_{2}(s) \in C^{1, \lambda}\left(\Gamma^{2}\right), \lambda \in(0,1]$, mo peшение задачи $\mathbf{U}$ существует, единственно и дается формулой (1), где $\mu(s)$ - решение системы (2), (3) в $C_{1 / 2}^{p}\left(\Gamma^{1}\right) \cap C^{0}\left(\Gamma^{2}\right), p=\min \{1 / 2, \lambda\}$, гарантированное леммой.

Решение задачи $\mathbf{U}$ удовлетворяет неравенству из п. (в) в определении класса $\mathbf{K}$ с показателем $\varepsilon=-1 / 2$.

\section{СПИСОК ЛИТЕРАТУРЫ}

[1] П. А. Крутицкий // Докл. РАН. 2001. Т. 376. № 1. С. 17-20.

Московский государственньй университет им. М. В. Ломоносова
Представлено М. И. Зеликиньм Принято редколлегией 23.07.2005 